\title{
Antoine Compagnon - Génétique, intertextualité et histoire littéraire
}

Entretien avec Pierre-Marc de Biasi et Anne Herschberg Pierrot

Antoine Compagnon, Pierre-Marc de Biasi et Anne Herschberg Pierrot

\section{OpenEdition}

\section{Journals}

Édition électronique

URL : https://journals.openedition.org/genesis/117

DOI : 10.4000/genesis. 117

ISSN : 2268-1590

\section{Éditeur :}

Presses universitaires de Paris Sorbonne (PUPS), Société internationale de génétique artistique littéraire et scientifique (SIGALES)

\section{Édition imprimée}

Date de publication : 20 juin 2010

Pagination : 55-57

ISBN : 978-2-84050-697-3

ISSN : $1167-5101$

\section{Référence électronique}

Antoine Compagnon, Pierre-Marc de Biasi et Anne Herschberg Pierrot, « Antoine Compagnon Génétique, intertextualité et histoire littéraire », Genesis [En ligne], 30 | 2010, mis en ligne le 17 mai 2013, consulté le 30 mars 2023. URL : http://journals.openedition.org/genesis/117 ; DOI : https:// doi.org/10.4000/genesis. 117 


\title{
Génétique, intertextualité et histoire littéraire Entretien avec Pierre-Marc de Biasi et Anne Herschberg Pierrot
}

\author{
Antoine Compagnon
}

\begin{abstract}
Antoine Compagnon, professeur au Collège de France, chaire de "Littérature française moderne et contemporaine : histoire, critique, théorie ", éditeur de Sodome et Gomorrhe de Proust dans la Pléiade, et spécialiste d' histoire littéraire et culturelle, a bien voulu répondre à nos questions sur ces domaines.
\end{abstract}

Pierre-Marc DE BiAsi - Comment un spécialiste de l' histoire littéraire, qui vient aussi de la théorie - ce n' est pas tout à fait indifférent - et qui a gardé un rapport fort avec ce moment formel, perçoit aujourd' hui les chances, l' intérêt et éventuellement les limites de la génétique dans le champ littéraire?

Antoine Compagnon - Cette relation n'est pas assez explorée ni exploitée. Prenons le cas de Proust. On sait mal tout ce qu'il a lu, on connaît insuffisamment sa culture. Encore aujourd'hui. Or les carnets, les brouillons, les cahiers peuvent contribuer à un enrichissement de ce genre de connaissance du texte. Il y a toutefois en amont quelque chose qui est plus absent encore : une philologie entendue dans un sens large et ambitieux, prenant acte d'un changement majeur de paradigme. Les philologues ne sont plus des sourciers, ils ne recherchent plus les sources biographiques ou bibliographiques comme ils le faisaient naguère.

Anne Herschberg Pierrot - Mais des traces intertextuelles.

A. C. - En effet. Et cela rend leur travail plus riche, mais plus compliqué. À l'époque où nous préparions l'édition de la Pléiade d'À la recherche du temps perdu, les choses avaient déjà changé. Les notes que nous avons faites pour cette édition, dans les années quatre-vingt, n'étaient plus celles des éditions traditionnelles se réclamant de l'histoire littéraire positiviste. L'intertextualité était passée par là. Il ne s'agissait plus seulement d'identifier, mais aussi de chercher à comprendre le travail du texte. Nous nous intéressions - en tout cas moi - au texte comme à une construction non linéaire, mais archéologique ou géologique, où les couches se transformaient, où les filons réapparaissaient sous la forme d'alluvions et d'allusions.

P.-M. DE B. - Une des contributions majeures de la génétique est d' avoir démontré que la question des sources d' un texte ne se limite pas aux traces observables dans sa version publiée, mais doit être posée à l'échelle de sa genèse : dans l'épaisseur des documents de travail, des carnets, des notes, des brouillons, où l' on peut suivre la manière dont l' écriture se trouve informée par une foule d'éléments autobiographiques, historiques, contextuels ou intertextuels qui progressivement s' intègrent à l'auvre au point de ne plus devenir repérables. Ces sources, pour la plupart, ne sont plus identifiables à l'échelle du texte définitif, ce qui ne les empêche pas d'avoir joué un rôle important, parfois décisif, dans la construction de l'cuvre, y compris dans certains cas par incompatibilité et par naufrage, ou encore par antagonisme, s'il s'agit d' une source conflictuelle contre laquelle le texte s'est écrit. Il s'agit là d'une voie importante pour la compréhension de l'écrivain et de son art : elle n'est guère praticable sans l' exploration des manuscrits.

A. C. - Oui, absolument. Nous avons assisté à une certaine convergence de la génétique et de l'intertextualité. Je me reconnais pleinement à cette intersection des études de genèse et des études intertextuelles, entendant celles-ci dans un sens large qui inclut l'histoire culturelle. C'est à ce confluent que je situe la plupart de mes travaux sur Proust depuis plus de deux décennies. Mais cela ne correspond pas au programme type de la génétique.

P.-M. DE B. - C' est quand même une des voies les plus pratiquées désormais par les chercheurs en génétique littéraire. 


\section{Génétique et réception}

P.-M. DE B. - L'autre question que j'aimerais vous poser porte sur les relations entre génétique et réception. La génétique, en principe, se trouve aux antipodes de la réception. Elle construit néanmoins l' hypothèse que la réception d' une ouvre est en grande partie construite par sa production, par la manière dont l'écrivain anticipe son destinataire, en inventant un lecteur qui n'existe pas encore. Là aussi, les brouillons, les documents de travail sont un espace dans lequel on voit cette stratégie se construire, quelquefois d'ailleurs explicitement, avec des notes de régie. La fabrication du lecteur est aussi une question cardinale de l' histoire littéraire, qui ne peut pas se résumer aux aléas d' une rencontre entre une auvre et un contexte historique, mais qui est aussi, pour une large part, l' effet de l' ouvre. Est-ce que vous n'estimez pas qu' il y a là matière pour l' histoire littéraire à un dialogue important avec les spécialistes des documents de genèse?

A. C. - À quels travaux pensez-vous ? Où trouvez-vous cette sorte de dialectique entre les études de réception et les études de genèse ?

P.-M. DE B. - Je pense par exemple aux études de genèse que nous menons à l'ITEM sur l'image du lecteur chez Balzac, Zola, Valéry, Joyce, Sartre, ou même Barthes, Michon... Pour ma part, je pense évidemment surtout à Flaubert : à la manière dont on peut suivre, par l'élaboration de sa poétique romanesque et par la mise en æuvre de cette poétique dans les brouillons, la construction d'un nouveau lecteur, à qui l'écrivain délègue la responsabilité de conclure sur le sens complexe et apparemment incohérent du récit. Ici, la génétique permet souvent de clarifier la dimension novatrice, anticipatrice, mais aussi intempestive, inactuelle, de l' ouvre : ce qui reste peu perceptible ou incompréhensible par son époque, mais qui construit ses chances en inventant un lecteur à venir, qui finira en effet par apparaître, une génération ou un siècle plus tard. C'était aussi le pari de Stendhal.

A. H. P. - C'est plus ou moins marqué selon les auteurs. Dans les brouillons de Flaubert, on trouve souvent la place du lecteur : "Faire croire au lecteur que... », etc. Chez
Proust, c'est peut-être moins évident, mais il y a quand même dans l'écriture une manière de programmer une réception.

A. C. - Il s'agit là d'une programmation intentionnelle de la réception de leurs œuvres par les écrivains, et ce n'est pas en général celle-là qui s'imposera à leurs lecteurs. J'ai toujours eu la conviction que les œuvres qui continuaient d'être lues par la postérité étaient de celles - non pas toutes, bien sûr - qui avaient échoué dans leur projet de s'adresser à un lecteur préconçu : il y a en elles une sorte de décalage productif entre leur lecteur intentionnel et leur écriture, ce qui leur donne prise sur l'avenir. Proust va plus loin que ses projets ; son roman leur est irréductible.

P.-M. DE B. - Mais, le plus souvent, ce décalage qui fait échouer l' euvre dans le présent est un gage délibéré sur l'avenir. C' est explicite chez Flaubert. Il dit : "j'écris pour aussi longtemps que la langue vivra ». L'œuvre s'écrit en refus de la mode et par anticipation de quelque chose de plus durable.

A. C. - Mais le lecteur qu'elle anticipe n'est pas pour autant celui dans lequel la postérité se reconnaîtra. Proust programme son lecteur à travers une théorie, celle du Temps retrouvé. Mais par la suite, ce sont plutôt d'autres raisons que sa doctrine qui ont attaché les lecteurs à son œuvre, d'autres chemins que ceux par lesquels Proust voulait les faire passer.

P.-M. DE B. - Dans le cas de Flaubert, je ne pensais pas à une doctrine ou à une théorie, d' ailleurs non exprimée dans l' euvre, mais vraiment à un style : à l'incarnation d' une poétique dans un récit, une poétique qui profile la place en creux d' un lecteur à venir, ses libertés, ses droits. L'Éducation sentimentale, de ce point de vue, est resté un texte illisible pour les contemporains de Flaubert : c'est devenu un siècle et demi plus tard un texte culte, pour des raisons que les manuscrits nous aident à comprendre.

A. C. - Chez Proust, le rôle de la théorie est complexe. Même si elle a été formulée pour commencer et si elle était indispensable, ensuite, comme tous les grands écrivains, Proust s'en écarte de plus en plus. C'est le travail du texte 
lui-même qui déconstruit la théorie qui a permis le texte, ce dont on retrouve trace dans la genèse. Par exemple, Proust, sous la forme de notes de régie, prévoit un peu partout à travers ses brouillons des amorces de la théorie finale du Temps retrouvé, pour relancer l'attente du lecteur, mais, au fur et à mesure de l'écriture, il efface ces pierres d'attente, soit qu'elles ne soient plus nécessaires, soit - c'est plutôt mon opinion - qu'elles ne correspondent plus à ce que le texte est devenu, plus subtil et irréductible à sa doctrine initiale. Il y a des indices de tout cela dans les cahiers.

\section{Génétique et histoire littéraire}

P.-M. DE B. - Je voudrais vous poser une dernière question, tout à fait ouverte, sur la façon dont vous définiriez les possibilités de collaborations dans l'avenir entre l' histoire littéraire et l'ensemble de ces recherches en génétique - sur les processus d'écriture, les manuscrits, les inédits, les notes, les documents de travail. Est-ce qu' il y a des zones sur lesquelles les réciprocités - on en a aperçu au passage quelques-unes - vous paraissent particulièrement précieuses ou fructueuses - indépendamment des questions d'édition?
A. C. - Encore une fois, prenons le cas de Proust. Il me semble que les travaux les plus féconds en ce moment sont ceux qui relèvent d'une histoire littéraire entendue dans le sens très vaste d'une histoire culturelle, archéologique et géologique des textes. Il est évident que cette histoire-là doit se fonder sur des études de genèse. Ce n'est qu'à partir de l'analyse fine des couches du texte que peuvent être repérées les résurgences géologiques ou archéologiques qui constituent le grain de la littérature, sa puissance d'allusion. Il me semble que la collaboration d'une perspective historique et d'un souci génétique est indispensable dans cette démarche. En tout cas, c'est comme cela que j'entends l'histoire littéraire aujourd'hui.

P.-M. DE B. - Comment en définiriez-vous les axes les plus forts ?

A. C. - L'histoire littéraire est une méthode contextuelle, une méthode d'exégèse contextuelle, et cette méthode a été fortement bousculée et enrichie par la prise en compte de tout ce qui relève du processus intertextuel, tel qu'il peut être repéré par les études de genèse. C'est cette méthode que j'ai essayé de pratiquer, et c'est vers elle que j'essaie d'orienter quelquesunes des meilleures thèses que j'ai eu la chance de diriger sur Proust. Contrairement à ce qu'on entend parfois, il reste beaucoup à faire en histoire littéraire ainsi comprise... 